\title{
Pesquisa Qualitativa na Saúde Bucal Coletiva: uma Análise Bibliográfica
} Qualitative Research in Public Oral Health: a Bibliographic Analysis

Gabriela Messa Schaarschmidt ${ }^{1}$, Fernando Valentim Bitencourt ${ }^{2}$, Alexandre Baumgarten ${ }^{3}$, Juliana Maciel de Souza ${ }^{4}$, Ramona Fernanda Ceriotti Toassi ${ }^{5}$

\begin{abstract}
Introduction: Qualitative researches are used in the study of subjects in which the results are not translated in numbers. This kind of research seeks to understand the process in which people construct meanings about what they leave and experience. Objective: To analyze the scientific production related to qualitative researches in public dental health. Methodology: A bibliographic analysis was conducted in the Virtual Health Library database with controlled descriptors. Results: A total of 55 articles were published between 1998 and 2013, with an increase trend in the number of articles published in this period ( 2 articles between 1998-1999 and 27 between 2010-2013). In more than half of the articles, the first author was Brazilian, followed by researchers from United Kingdom, Canada and USA. Most of the authors were from universities. Articles were mostly published in journals of public dental health and dentistry, and the subjects of study, which included dentists, health professionals, patients/health services users and dental students were submitted to interview. The methods of data interpretation used in most of the studies were thematic analysis and content analysis. These researches focused on the themes such as Family Health Strategy, Dental Health Policy, and Dental Education/Teaching. Conclusion: In order to consolidate qualitative research in public dental health, it is necessary and include an increase in the number of articles published with this methodology, increase funding, as well as publications linked to academic papers characterized by methodological rigor articulated to the necessary epistemological substantiation (indissociation theory and technique).
\end{abstract}

Keywords: Qualitative research. Public health. Oral health. Dentistry.

\section{Resumo}

Introdução: Pesquisas qualitativas são utilizadas quando o objeto de pesquisa exige respostas que não se traduzem em números. Buscam entender o processo pelo qual as pessoas constroem significados sobre o que vivenciam e experenciam. Objetivo: Analisar a produção científica em relação à pesquisa qualitativa na saúde bucal coletiva. Metodologia: Uma análise bibliográfica foi conduzida na base de dados da Biblioteca Virtual em Saúde com descritores controlados. Resultados: Foram avaliados 55 artigos publicados entre 1998-2013, observando-se uma tendência de aumento no número de publicações no período (1998-1999: 2 artigos; 2010-2013: 27 artigos). O primeiro autor, em mais da metade dos artigos, era do Brasil, seguido pelo Reino Unido, Canadá e EUA. Esses autores estavam vinculados principalmente a universidades. Os artigos, em sua maioria, foram publicados em periódicos de saúde pública e odontologia (destaque para Revista Ciência \& Saúde Coletiva), utilizando a entrevista e incluindo cirurgiões-dentistas, profissionais da saúde, pacientes/usuários de serviços de saúde e estudantes de odontologia. A análise temática e a análise de conteúdo foram os métodos de interpretação mais utilizados nos estudos. Destacam-se as pesquisas qualitativas com temáticas voltadas à Estratégia de Saúde da Família, Política de Saúde Bucal e Educação/Ensino em Odontologia.
Conclusão: Desafios importantes devem ser superados para a consolidação de pesquisas qualitativas na saúde bucal coletiva, o que inclui crescimento no número de artigos publicados com essa metodologia, maior financiamento, além de publicações vinculadas a trabalhos acadêmicos caracterizados pelo rigor metodológico articulado ao necessário fundamento epistemológico (indissociação teoria e técnica).

Descritores: Pesquisa qualitativa. Saúde pública. Saúde bucal. Odontologia

${ }^{1}$ Cirurgiã-dentista. Universidade Federal do Rio Grande do Sul (UFRGS). Porto Alegre, Rio Grande do Sul, Brasil - Porto Alegre-RS, Brasil).

${ }^{2}$ Bolsista de Iniciação Científica. Universidade Federal do Rio Grande do Sul (UFRGS). Porto Alegre, Rio Grande do Sul, Brasil.

${ }^{3}$ Cirurgião-dentista. Mestrando do Programa de Pós-Graduação em Epidemiologia. Universidade Federal do Rio Grande do Sul (UFRGS). Porto Alegre, Rio Grande do Sul, Brasil.

${ }^{4}$ Mestre em Ensino na Saúde. Programa de Pós-Graduação em Ensino na Saúde da Universidade Federal do Rio Grande do Sul (UFRGS). Porto Alegre, Rio Grande do Sul, Brasil.

${ }^{5}$ Doutora em Educação. Professora do Departamento de Odontologia Preventiva e Social da Faculdade de Odontologia e do Programa de Pós-Graduação em Ensino na Saúde da Faculdade de Medicina. Universidade Federal do Rio Grande do Sul (UFRGS). Porto Alegre, Rio Grande do Sul, Brasil.

Correspondência: Dra. Ramona F. C. Toassi

Endereço: Faculdade de Odontologia da UFRGS, Departamento de Odontologia Preventiva e Social. Rua Ramiro Barcelos, 2492. Porto Alegre, Rio Grande do Sul, Brasil 90035-003. E-mail: ramona.fernanda@ufrgs.br

\section{Introdução}

No campo da saúde, pesquisas com abordagem qualitativa utilizam-se da concepção trazida das Ciências Humanas de que não se busca estudar um fenômeno em si, mas sim entender o processo pelo qual as pessoas constroem significados sobre esses fenômenos. $\mathrm{O}$ fenômeno refere-se a tudo o que os seres humanos vivem e experimentam e é pelo significado desses fenômenos que as pessoas organizam suas vidas e cuidados com a saúde (TURATO, 2005; TITCHEN; HOBSON, 2015; BOGDAN; BIKLEN, 1998). Os pesquisadores que desenvolvem pesquisas qualitativas estudam os fenômenos no ambiente natural do sujeito, buscando dar sentido ou interpretar fenômenos nos termos das significações (DENZIN; LINCOLN, 2006).

Cada vez mais difundida na saúde coletiva, a pesquisa qualitativa é utilizada quando o objeto de pesquisa exige respostas que não se traduzem em números, sendo estruturada na experiência, na vivência, no senso comum e na ação. Leva em conta a singularidade do indivíduo, por meio de sua subjetividade (MINAYO, 2012), ou seja, examina a compreensão subjetiva das pessoas a respeito de sua vida diária (POPE; MAYS, 2009). A finalidade é a 
crítica e a transformação da realidade; compreensão e reconstrução (MIGUEL; REIBNITZ JÚNIOR; PRADO, 2007).

Considerada pela tradição quantitativa como uma pesquisa não objetiva (SERAPIONI, 2005), a pesquisa qualitativa foi se destacando na saúde coletiva e, embora não considerada hegemônica e com vários desafios para sua afirmação plena, vem merecendo atenção no campo (BOSI, 2012).

Minayo (2010) entende que o fundamental é a compreensão de que tanto a abordagem quantitativa quanto a qualitativa tem seu papel, seu lugar, sua adequação e ambas podem conduzir a resultados importantes sobre a realidade social, não havendo sentido atribuir prioridade de um sobre o outro.

Para a odontologia, historicamente, o conhecimento produzido tem se fundamentado no paradigma quantitativo, por conta do tecnicismo do ato odontológico (MIGUEL; REIBNITZ JÚNIOR; PRADO, 2007). A pesquisa qualitativa, nesse contexto, representa um novo caminho a ser trilhado pela odontologia, possibilitando o planejamento de ações éticas e humanizadas em saúde coletiva e trazendo melhores resultados à população em razão da profundidade de conhecimento que seus dados podem gerar (GARBIN et al., 2013).

Apesar dessa perspectiva promissora, estudo de Masood et al. (2011) mostrou a baixa qualidade dos estudos qualitativos em odontologia e Celeste, Moyses e Broadbent (2016) identificaram que as publicações qualitativas em saúde bucal coletiva no contexto geral da área em nível mundial são quase nulas. Há de se considerar, ainda, a discriminação de publicações com tal abordagem de pesquisa, mesmo quando possui boa qualidade e alto impacto (GREENHALGH et al., 2016).

Conhecer a produção de pesquisas qualitativas em saúde bucal pode contribuir para a avaliação da qualidade e pertinência dessas pesquisas, bem como pode reforçar seu significado no contexto de trabalho do cirurgião-dentista clínico, que atua no cuidado de pessoas com sentimentos e subjetividades singulares. Assim o objetivo do presente estudo é analisar a produção científica em relação à pesquisa qualitativa na saúde bucal coletiva.

A intenção que o moveu foi a de apresentar informações (corte descritivo) sobre a produção científica da pesquisa qualitativa na área, trazendo essa discussão à tona e instigando a realização de novas pesquisas que possam avançar em análises de cunho epistemológico.

\section{Materiais e Métodos}

O estudo foi aprovado pela Comissão de Pesquisa da Faculdade de Odontologia da Universidade Federal do Rio Grande do Sul (projeto $n^{\circ} 27085$ ).

Caracteriza-se como uma análise bibliográfica conduzida em março de 2014, na base de dados bibliográficos da BVS - Biblioteca Virtual em Saúde (<http://www.bireme.br>), a qual inclui as seguintes fontes de informação: LILACS (Literatura Latino-Americana e do Caribe em Ciências da Saúde), IBECS (Índice Bibliográfico Espanhol de Ciências da Saúde), MEDLINE (Medical Literature Analysis and Retrieval System Online), Biblioteca Cochrane e SciELO (Scientific Electronic Library Online).

A identificação dos descritores controlados para a busca foi realizada junto ao DeCS-Descritores em Ciências da Saúde. Foram utilizados os descritores controlados: pesquisa qualitativa / saúde pública / saúde coletiva / saúde bucal / odontologia e qualitative research/ public health/ oral health/ dentistry. Esses descritores foram cruzados acrescidos do operador booleano 'AND' nas combinações: - pesquisa qualitativa AND saúde pública AND saúde bucal - pesquisa qualitativa AND saúde pública AND odontologia - pesquisa qualitativa AND saúde coletiva AND saúde bucal
- pesquisa qualitativa AND saúde coletiva AND odontologia - qualitative research AND public health AND oral health

- qualitative research AND public health AND dentistry

Não foi especificado um período limite de tempo. No campo de pesquisa deveriam constar os descritores no título, resumo ou assunto dos artigos.

Como critérios de inclusão, os artigos deveriam ter sido publicados em português, inglês ou espanhol envolvendo a temática estudada 'pesquisa qualitativa na saúde bucal coletiva'. Foram excluídos da seleção, teses, monografias, editoriais, revisões da literatura, artigos de bases duplicadas ou que não estivessem incluídos nos critérios de inclusão.

Todos os pesquisadores participaram da seleção dos artigos, o que envolveu um treinamento prévio do grupo, baseando-se nos critérios de inclusão e exclusão, previamente estabelecidos.

Para a análise dos artigos, foi construída uma planilha eletrônica com as seguintes variáveis: (1) número de publicações por ano; (2) número de autores, região geográfica de afiliação dos autores e tipo de instituição vinculada; (3) fonte de financiamento e vinculação com trabalhos acadêmicos; (4) periódico de publicação/assunto/Qualis; (5) tipo de pesquisa qualitativa; (6) técnicas de coleta de dados; (7) métodos de análise de dados; (8) recortes temáticos; (9) participantes das pesquisas; (10) aspectos éticos.

O programa Microsoft Excel versão 2010 (Microsoft@ Office) foi utilizado para a construção da planilha eletrônica e cálculo das frequências e médias.

\section{Resultados}

Foram encontrados 281 trabalhos com a temática pesquisa qualitativa na saúde bucal coletiva. Destes, 41 foram excluídos por se tratarem de teses ou monografias (4 duplicadas) e 79 artigos por estarem duplicados entre as bases consultadas. Após as exclusões, 161 artigos foram selecionados, os quais foram obtidos na íntegra para análise. Após a leitura, 12 foram excluídos por serem uma revisão da literatura, 1 por ser ensaio, 2 editoriais e 91 por não se referirem à temática da pesquisa (Figura 1).

Ao final, foram avaliados 55 artigos na base da BVS voltados à pesquisa qualitativa na saúde bucal coletiva.

Os 55 artigos foram publicados entre 1998 e 2013, observando-se um número maior de publicações no período de 2010 a 2013 (49,1\%). O número de autores por artigo variou de um a sete (média de 3,4 autores por artigo), totalizando 187 autores nos 55 artigos. A maior parte dos artigos analisados apresentou entre três e quatro autores (49,1\%). Brasil (52,8\%), Reino Unido (12,8\%), Canadá $(9,1 \%)$ e EUA $(9,1 \%)$ foram os países em que houve um maior número de primeiros autores vinculados aos artigos produzidos sobre a temática estudada. Esses primeiros autores apresentavam, em sua maioria, vínculo com universidades. A Universidade de São Paulo (MATOS; TOMITA, 2004; LENZI et al., 2010; MUNKEVIZ; PELICIONI, 2010; ANDRADE et al., 2011), a Universidade Federal de Minas Gerais (VARGAS; PAIXÃO, 2005; SANTIAGO; WERNECK; FERREIRA, 2009; VASCONCELOS; CARDOSO; ABREU, 2010) e a Universidade Federal do Rio Grande do Sul (DE MARCHI et al., 2012; NEVES; PRETTO; ELY, 2013; TOASSI et al., 2013) foram as Instituições de Ensino Superior que tiveram um número maior de primeiros autores nos artigos identificados (Tabela 1).

A análise da região geográfica de afiliação dos 29 primeiros autores brasileiros revelou uma distribuição desigual entre as cinco regiões do país, sendo que as regiões Norte e Centro-Oeste não tiveram representação nos artigos avaliados. A região Sudeste 
$(41,4 \%)$ foi a que mais esteve representada nos artigos avaliados, seguida da região Sul (31\%) e da região Nordeste $(27,6 \%)$.
A vinculação dos artigos com trabalhos acadêmicos foi apresentada em três publicações brasileiras (Trabalho de Conclusão de Curso de Aperfeiçoamento, Dissertação de Mestrado e Tese de Doutorado).

Figura 1 - Fluxograma com a combinação dos descritores selecionados e sistematização do processo de seleção dos artigos.

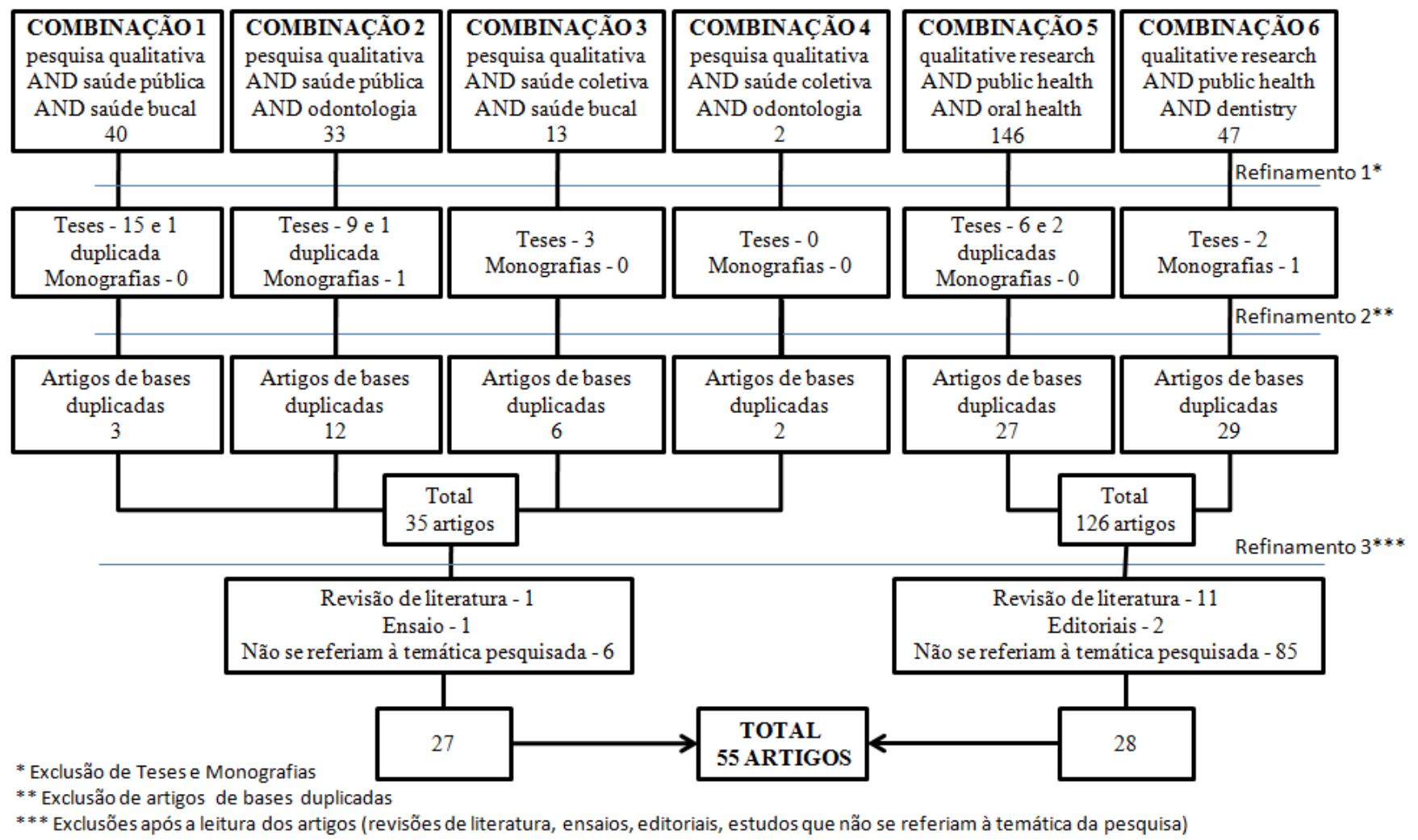

Dois primeiros autores destacaram-se nesta pesquisa bibliográfica por apresentarem dois artigos publicados sobre a temática da pesquisa qualitativa na saúde bucal coletiva no período estudado - Luciane Maria Pezzato, vinculada à Pontifícia Universidade Católica de Campinas, Brasil (PEZZATO, L.M.; L'ABBATE, 2011; 2012) e Christophe Bedos, da McGill University, Canadá (BEDOS; LEVINE; BRODEUR, 2009; BEDOS et al., 2013).

Em quatro publicações brasileiras houve a parceria de autoria entre Universidade e Sistema Único de Saúde (SUS). A Residência Multiprofissional em Saúde da Família e Comunidade apareceu vinculada a um dos 55 artigos.

A fonte de financiamento das pesquisas foi informada em $29 \%(n=16)$ dos artigos. As agências de fomento citadas nos trabalhos foram: Conselho Nacional de Desenvolvimento Científico, Tecnológico (CNPq), Fundação de Amparo à Pesquisa do Rio Grande do Sul (FAPERGS) e Fundação de Amparo à Pesquisa do Estado de São Paulo (FAPESP), Canadian Institutes of Health Research, Quebec Research Fund, The Ben Walton Trust, University of Otago, NHS, University of Missouri-kansas City, School of Dentistry Rinehart Foundation, SESPO, Northern Regional Health Authority, NSW Health, Centre for Oral Health Strategy, Oral Health Promotion Demonstration Program Grants Scheme, Queens Nursing Institute Scotland, FRSQ Network Heath Research, National Health, University of Tasmania.
Em relação ao periódico de publicação, a revista com maior número de artigos publicados com a temática da pesquisa qualitativa na saúde bucal coletiva foi a Ciência \& Saúde Coletiva $(9,1 \%)$, seguida por Cadernos de Saúde Pública $(7,3 \%)$ e British Dental Journal $(7,3 \%)$. Os dois primeiros são periódicos cujos assuntos estão relacionadas à saúde pública e o último à odontologia (Tabela 2). São periódicos com classificação Qualis B1 para a saúde coletiva e odontologia - Ciência \& Saúde Coletiva), A2 para a saúde coletiva e odontologia - Cadernos de Saúde Pública) e B1 para odontologia British Dental Journal). Cinco periódicos (um de medicina, três de saúde pública e um de odontologia) não apresentavam classificação Qualis no momento da coleta de dados. Em quase metade dos artigos avaliados, a informação apresentada sobre o tipo de estudo, referiase apenas que se tratava de pesquisa/estudo/método/abordagem/corte qualitativa (o)' (43,7\%). Quando havia a definição do tipo de pesquisa qualitativa realizada, o estudo de caso foi o mais observado (9\%), seguido pela pesquisa exploratória $(7,3 \%)$ e pesquisa qualitativa descritiva exploratória $(5,6)$. A entrevista foi a técnica de coleta de dados mais utilizada nos artigos qualitativos avaliados $(58,2 \%)$, seguida pela associação de técnicas $(16,5 \%)$ e grupo focal $(7,2 \%)$. O método de análise de dados mais frequente foi a análise temática $(32,8 \%)$ e a análise de conteúdo $(25,5 \%)$. Dos 14 estudos que utilizaram a análise de conteúdo, nove 
Tabela 1 - Distribuição dos artigos analisados segundo o ano de publicação, número de autores por artigo, país e vínculo de afiliação do primeiro autor.especificaram a análise de conteúdo de Bardin.

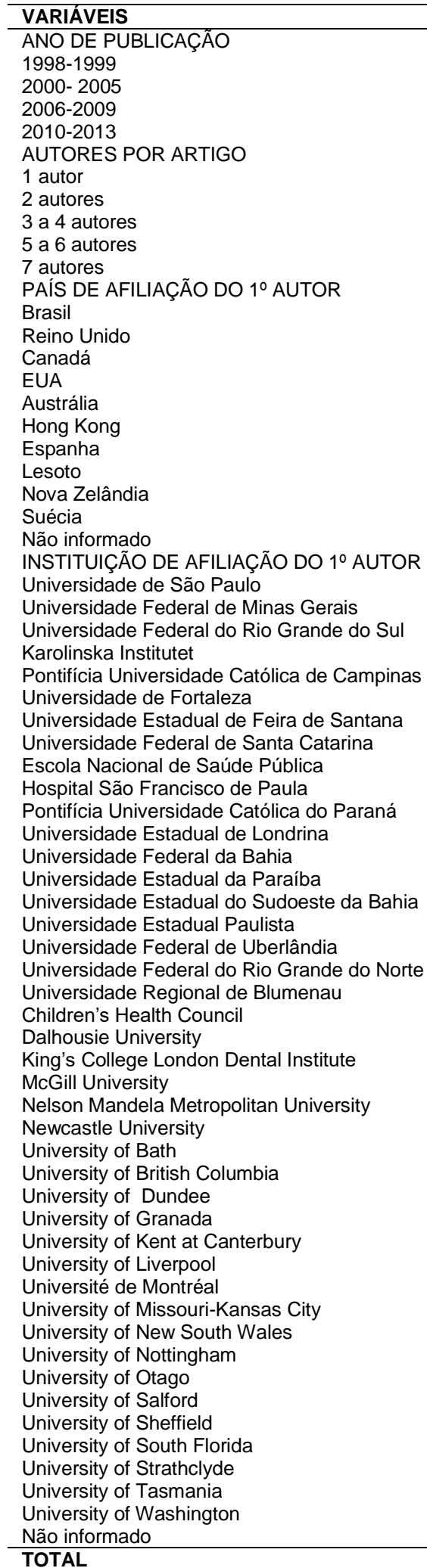

n $\%$

23,6

$29 \quad 52,8$

$7 \quad 12,8$

59,1

$5 \quad 9,1$

23,6

23,6

$1 \quad 1,8$

$1 \quad 1,8$

$1 \quad 1,8$

$\begin{array}{ll}1 & 1,8 \\ 1 & 1,8\end{array}$

47,3

$$
7,3
$$

5,6 trabalhos não informaram o método de interpretação utilizado. Nos recortes temáticos dos artigos observa-se um número maior de pesquisas qualitativas voltadas à Estratégia de Saúde da Família (16,4\%), Política de Saúde Bucal (16,4\%) e Educação/Ensino em Odontologia (11\%) (Tabela 2).

$\begin{array}{ll}2 & 3,6 \\ 5 & 9,1 \\ 21 & 38,2 \\ 27 & 49,1 \\ & \\ 2 & 3,6 \\ 15 & 27,3 \\ 27 & 49,1 \\ 9 & 16,4 \\ 2 & 3,6\end{array}$

Dos 55 artigos, dois referiam-se a pesquisas de caráter documental exclusivo. Todos os demais 53 artigos tratavam de pesquisas realizadas com seres humanos. Nesses estudos, a amostragem qualitativa foi definida como proposital, intencional ou deliberada, com critérios estabelecidos pelos pesquisadores e apresentados nos artigos. O Quadro 1 apresenta as informações sobre os participantes das pesquisas.

O uso de softwares para apoiar a análise dos dados qualitativos foi citado em 8 dos 55 artigos avaliados. Seis estudos utilizaram o software NVivo e dois o Visual Qualitative Data Analysis (ATLAS.ti).

Em relação aos aspectos éticos, $72,7 \%$ dos artigos relataram a aprovação das pesquisas em Comitês de Ética. Doze artigos não apresentaram informação sobre os aspectos éticos e três mencionaram apenas que os sujeitos de pesquisa autorizaram previamente a realização de entrevistas.

Tabela 2 - Distribuição dos artigos segundo o periódico de publicação, tipo de pesquisa qualitativa, técnica de coleta de dados, método de análise de dados e recortes temáticos das pesquisas.

\begin{tabular}{lcc}
\hline VARIÁVEIS & n & $\%$ \\
\hline PERIÓDICO DE PUBLICAÇÃO/ASSUNTO & & \\
Ciência \& Saúde Coletiva/Saúde Pública & 9,1 \\
Cadernos de Saúde Pública/ Saúde Pública & 4 & 7,3 \\
British Dental Journal/ Odontologia & 4 & 7,3 \\
Arquivos em Odontologia/Odontologia & 2 & 3,7 \\
Interface: comunicação, saúde e educação/ Saúde Pública e & 2 & 3,7 \\
Educação & 2 & 3,7 \\
International Journal of Dental Hygiene/Odontologia & 2 & 3,7 \\
International Dental Journal/ Odontologia & 2 & 3,7 \\
Pesquisa Brasileira em Odontopediatria e Clínica Integrada/ & & \\
Odontologia & 2 & 3,7 \\
Physis (Rio de Janeiro)/ Saúde Pública & 2 & 3,7 \\
Saúde e Sociedade/Saúde Pública & 1 & 1,8 \\
Acta Odontologica Scandinavica/Odontologia & 1 & 1,8 \\
Australian Journal of Rural Health/Enfermagem e Saúde Pública & 1 & 1,8 \\
BMC Health Services Research/Pesquisa em Serviços de Saúde & 1 & 1,8 \\
BMC Public Health/Saúde Pública & 1 & 1,8 \\
Cogitare enfermagem/ Enfermagem & 1 & 1,8 \\
Community Dental Health/Odontologia e Saúde Pública & 1 & 1,8 \\
Community Dental Oral Epidemiology/Odontologia & 1 & 1,8 \\
Eastern Mediterranean Health Journal/Medicina & 1 & 1,8 \\
European Journal of Dental Education/Odontologia & 1 & 1,8 \\
Health Promotion Journal of Australia/Saúde Pública & 1 & 1,8 \\
Journal Community Health/Saúde Pública e Prestação de & 1
\end{tabular}

Journal Community Health/Saúde Pública e Prestação de 1 1,8 cuidados em saúde

Journal of Cross-Cultural Gerontology/Geriatria

Journal of Dental Research/ Odontologia

Journal of Public Health Medicine/Saúde Pública

Journal of the Health Sciences Institute/Medicina

Maternal and Child Health Journal/Perinatologia

Medicina (Ribeirão Preto)/ Medicina

New Zealand Dental Journal/ Odontologia

Ortho Science: orthodontics science and practice/ Odontologia 11,8

Odontologia clínico-científica/Odontologia 11,8

Pediatric Dentistry/ Odontologia 11,8

Revista Brasileira de Crescimento e Desenvolvimento Humano/ 1 1,8

Saúde Pública e Pediatria

Revista de APS/Prestação de Cuidados em Saúde 1,8

Revista de Saúde Pública/Saúde Pública 11,8

Revista de Odontologia da UNESP (Impresso)/ Odontologia 11,8

Rural and Remote Health/Saúde Pública 11,8

Social Science \& Medicine/ Sociologia e Prestação de cuidados $1 \quad 1,8$ 


\begin{tabular}{|c|c|c|}
\hline VARIÁVEIS & $\mathbf{n}$ & $\%$ \\
\hline \multicolumn{2}{|l|}{ TIPO DE PESQUISA QUALITATIVA } & 1,8 \\
\hline Pesquisa/estudo/método/abordagem/corte qualitativa (o) & 24 & 43,7 \\
\hline Pesquisa quantitativa e qualitativa & 7 & 12,8 \\
\hline Estudo de caso & 5 & 9,0 \\
\hline Pesquisa exploratória & 4 & 7,3 \\
\hline Pesquisa qualitativa de caráter descritivo exploratório & 3 & 5,6 \\
\hline Pesquisa descritiva & 2 & 3,6 \\
\hline Pesquisa etnográfica & 2 & 3,6 \\
\hline Pesquisa ação-intervenção & 1 & 1,8 \\
\hline Pesquisa empírica-exploratória & 1 & 1,8 \\
\hline Pesquisa exploratória documental com abordagem qualitativa & 1 & 1,8 \\
\hline Pesquisa qualitativa retrospectiva & 1 & 1,8 \\
\hline Pesquisa qualitativa convergente-assistencial & 1 & 1,8 \\
\hline Estudo de abordagem qualitativa valendo-se da técnica Delphi & 1 & 1,8 \\
\hline $\begin{array}{l}\text { Não informado } \\
\text { TÉCNICA DE COLETA DE DADOS }\end{array}$ & 2 & 3,6 \\
\hline Entrevista & 32 & 58,2 \\
\hline Associação de técnicas & 9 & 16,5 \\
\hline Grupo focal & 4 & 7,2 \\
\hline Questionário & 3 & 5,5 \\
\hline Análise documental & 2 & 3,6 \\
\hline Diário & 2 & 3,6 \\
\hline Mapas de conversação & 1 & 1,8 \\
\hline Narrativas por telefone & 1 & 1,8 \\
\hline Não informada & 1 & 1,8 \\
\hline \multicolumn{3}{|l|}{ MÉTODO DE ANÁLISE DE DADOS } \\
\hline Análise Temática & 18 & 32,8 \\
\hline Análise de Conteúdo & 14 & 25,5 \\
\hline Teoria Fundamentada nos Dados & 4 & 7,3 \\
\hline Discurso do Sujeito Coletivo & 3 & 5,5 \\
\hline Método Hermenêutico-Dialético & 2 & 3,6 \\
\hline Análise Institucional & 2 & 3,6 \\
\hline Análise Ideográfica e Namotética & 1 & 1,8 \\
\hline Método Tesch & 1 & 1,8 \\
\hline Técnica Delphi & 1 & 1,8 \\
\hline Não informado & 9 & 16,3 \\
\hline \multicolumn{3}{|l|}{ RECORTES TEMÁTICOS } \\
\hline Estratégia de Saúde da Família & 9 & 16,4 \\
\hline Política de Saúde Bucal & 9 & 16,4 \\
\hline Educação/ensino em odontologia & 6 & 11,0 \\
\hline Enfermagem e saúde bucal & 3 & 5,6 \\
\hline Promoção da saúde & 3 & 5,6 \\
\hline Perda dentária & 2 & 3,6 \\
\hline Acesso aos cuidados de saúde bucal & 2 & 3,6 \\
\hline Atendimento odontológico na zona rural & 2 & 3,6 \\
\hline Diários como ferramenta de intervenção & 2 & 3,6 \\
\hline Saúde bucal e agentes comunitários de saúde & 2 & 3,6 \\
\hline Saúde bucal e assistência social & 2 & 3,6 \\
\hline Atendimento no serviço público e no serviço privado & 1 & 1,8 \\
\hline Avaliação de programa odontológico & 1 & 1,8 \\
\hline Biossegurança & 1 & 1,8 \\
\hline Câncer de boca & 1 & 1,8 \\
\hline Dor de dente & 1 & 1,8 \\
\hline Doença periodontal & 1 & 1,8 \\
\hline Ética/Bioética & 1 & 1,8 \\
\hline Fluoretação de águas & 1 & 1,8 \\
\hline Hálito e relações sociais & 1 & 1,8 \\
\hline Saúde bucal e baixa renda & 1 & 1,8 \\
\hline Saúde bucal e mídia impressa & 1 & 1,8 \\
\hline Saúde bucal e parto prematuro & 1 & 1,8 \\
\hline Saúde bucal e práticas populares de cuidado & 1 & 1,8 \\
\hline TOTAL & 55 & 100,0 \\
\hline
\end{tabular}

Quadro 1 - Informações sobre os participantes de pesquisa

\begin{tabular}{|c|c|}
\hline $\begin{array}{ll}\text { PARTICIPANTES } & \text { DAS } \\
\text { PESQUISAS } & \end{array}$ & DESCRIÇÃO \\
\hline Número de participantes & $\begin{array}{l}\text { Variação de } 5 \text { a } 67 \text { entrevistados } \\
(3 \text { artigos não apresentaram essa } \\
\text { informação) }\end{array}$ \\
\hline População da pesquisa & $\begin{array}{l}\text { Cirurgiões-dentistas }(n=10) \\
\text { Pacientes/usuários dos serviços de } \\
\text { saúde }(n=8) \\
\text { Estudantes de odontologia }(n=4) \\
\text { Profissionais da saúde }(n=3) \\
14 \text { estudos incluíram mais de um grupo } \\
\text { de participantes de pesquisa }\end{array}$ \\
\hline $\begin{array}{l}\text { Método de amostragem (definição } \\
\text { do número de participantes) }\end{array}$ & $\begin{array}{l}13 \text { estudos citaram a amostragem por } \\
\text { saturação } \\
24 \text { estudos determinaram o número de } \\
\text { participantes pesquisados a partir dos } \\
\text { critérios de inclusão estabelecidos } \\
14 \text { estudos não apresentaram clareza } \\
\text { no critério estabelecido para a } \\
\text { determinação do número de } \\
\text { participantes da pesquisa }\end{array}$ \\
\hline
\end{tabular}

\section{Discussão}

O presente estudo propôs-se a realizar um estudo bibliográfico da produção científica em relação à pesquisa qualitativa na saúde bucal coletiva. Sua relevância baseia-se nas contribuições que a pesquisa qualitativa pode possibilitar à área da saúde (GARBIN et al., 2013; GONÇALVES; MENASHE, 2014) e, em especial, para a saúde pública (JACK, 2006).

O número de artigos identificados sobre a temática estudada foi de 55. Esses artigos foram publicados entre os anos de 1998 e 2013. É importante considerar que dezembro de 2013 foi estabelecido como o período final de busca na presente pesquisa. De 2010 a 2013 houve um aumento quantitativo nessa produção comparado aos períodos de 1998-1999 e 2000-2009. Apesar desse crescimento, o número de artigos identificados ainda é baixo e parece não ter acompanhado o aumento verificado, de modo geral, no número de publicações no campo da saúde coletiva (CANESQUI, 2012; LUZ; MATTOS, 2010) e nas publicações da odontologia relacionadas à saúde pública/saúde coletiva (DIAS; NARVAI; RÊGO, 2008; NADANOVSKY, 2006).

Estudos de revisão de literatura reforçam a fragilidade das pesquisas qualitativas na odontologia expresso pelo baixo número de artigos publicados (NEWTON. 2000; AMORIM et al., 2007; CELESTE; WARMLING, 2014). Pesquisas abrangendo conteúdos técnicos e de cunho eminentemente profissional, como levantamentos epidemiológicos, programas de saúde bucal e fluoretação (AMORIM et al., 2007) ou voltados à epidemiologia, são dominantes na odontologia (CELESTE; WARMLING, 2014).

Observa-se, assim, que a contribuição das pesquisas de abordagem qualitativa para a base de evidências da odontologia, tem sido discreta. Historicamente o conhecimento produzido na odontologia tem se fundamentado no paradigma quantitativo, que tem sido hegemônico na produção do conhecimento científico no último século e está fundado na capacidade de explicar a relação causaefeito. O modelo cirúrgico restaurador trouxe poucas respostas às necessidades acumuladas da população em programas de assistência odontológica, e o planejamento das ações baseadas em dados quantitativos respondeu parcialmente às necessidades das pessoas envolvidas (MIGUEL; REIBNITZ JÚNIOR; PRADO, 2007).

A utilização da metodologia qualitativa nas pesquisas tem, nesse contexto, potencial para trazer à odontologia conhecimento em relação à percepção das pessoas, proporcionando uma forma mais 
abrangente de compreensão de suas crenças, saberes e atitudes, bem como oferecer maior profundidade e flexibilidade metodológica do que os métodos quantitativos de pesquisa (STEWART, 2008).

$\mathrm{Na}$ perspectiva da saúde bucal coletiva, a pesquisa qualitativa torna-se cada vez mais relevante para a compreensão dos fatores sociais, culturais e econômicos que afetam o estado de saúde e cuidados de saúde do indivíduo e da população como um todo. Seu uso permite que os pesquisadores respondam importantes questões de pesquisa que são difíceis de resolver satisfatoriamente usando métodos quantitativos sozinhos (BOWERE; SCAMBLER, 2007; GEORGE; KRUGER; TENNANT , 2012).

Com relação às temáticas estudadas nos 55 artigos voltados à pesquisa qualitativa na saúde bucal coletiva identificados neste estudo, a 'Estratégia de Saúde da Família' junto com a 'política de saúde bucal' foram as que mais se destacaram. Estudos bibliométricos realizados no Brasil analisando a produção científica das ciências sociais e humanas em saúde em periódicos de saúde coletiva/saúde pública (NADANOVSKY, 2006) e em saúde bucal coletiva com periódicos da saúde coletiva (CELESTE; WARMLING, 2014), também mostraram a ênfase na temática das políticas de saúde.

A opção pelo tema das políticas de saúde pode ser justificada pela possibilidade das pesquisas qualitativas serem mais informativas do que as quantitativas, aplicando métodos desenvolvidos nas ciências sociais, permitindo uma descrição minuciosa dos desafios emergenciais da saúde pública (STOTO; NELSON; KLAIMAN, 2012) e o desenvolvimento de estratégias de intervenção.

Nos artigos avaliados nesta pesquisa, ressalta-se, além do número limitado de artigos encontrados, a presença de lacunas importantes quanto à apresentação de aspectos éticos, definição conceitual do entendimento sobre o que seja uma pesquisa de 'dimensão qualitativa' com o necessário fundamento epistemológico que sustente as escolhas metodológicas e a clareza em questões relativas ao desenvolvimento das pesquisas qualitativas (método de análise/interpretação, critérios para determinação do número de participantes das pesquisas).

Análises de Cyrino (2014) e Knauth e Leal (2014) mostraram que, se por um lado, as metodologias qualitativas têm tido aceitação e penetração nas pesquisas da área da saúde, por outro, têm revelado problemas e dificuldades na qualidade do que tem sido produzido, destacando-se a fragilidade dos trabalhos quanto à consistência teórico-metodológica, ausência de referencial teórico, apresentação de resultados quantificados com muita descrição e pouca interpretação dos dados empíricos, e limitada contextualização do objeto e campo do estudo.

Tais fragilidades também foram observadas no estudo de avaliação da qualidade das pesquisas qualitativas na odontologia entre 1999 e 2006 realizado por Masood et al. (2011). Os resultados identificaram $48 \%$ dos artigos com foco na pesquisa em saúde bucal coletiva, 35\% que forneceram pouca ou nenhuma informação sobre o processo de análise, metade não forneceu informações sobre os aspectos éticos, $30 \%$ dos trabalhos não apresentaram a justificativa dos métodos escolhidos e metade dos trabalhos não discutiram porque o tamanho da amostra era adequado à metodologia proposta.

Acrescenta-se, ainda, o papel fundamental das Instituições de Ensino Superior (graduação e pós-graduação), agências e institutos de fomento de pesquisa no desenvolvimento e apoio a linhas de pesquisa na saúde que contemplem abordagens qualitativas de construção do conhecimento. Poucos foram os estudos encontrados nesta pesquisa que mostraram fonte de financiamento e vinculação das pesquisas qualitativas com trabalhos acadêmicos.

\section{Conclusão}

A análise bibliográfica da produção científica em relação à pesquisa qualitativa na saúde bucal coletiva a partir da busca na base de dados da Biblioteca Virtual em Saúde com descritores controlados (pesquisa qualitativa, saúde coletiva, saúde pública, saúde bucal, odontologia) mostrou uma tendência de crescimento no número de publicações de 1998 a 2013 (de 2 artigos entre 1998-1999 para 27 entre 2010-2013). Esse aumento numérico, entretanto, é discreto diante do crescente número de revistas científicas e dos programas de pós-graduação tanto na área da saúde coletiva quanto na odontologia.

Para além dos números, que ainda são escassos, os resultados expressam desafios voltados a consolidação das pesquisas qualitativas na saúde bucal coletiva. Tais desafios incluem um maior financiamento para a realização dessas pesquisas além de publicações vinculadas a trabalhos acadêmicos na graduação e pósgraduação caracterizados pelo rigor metodológico articulado ao necessário fundamento epistemológico (indissociação teoria e técnica).

Este estudo assume como limitações a intencionalidade da opção de busca de publicações a partir de descritores controlados junto ao DECs que abrangessem de modo específico a pesquisa qualitativa na saúde bucal coletiva, o que pode ter restringindo a identificação de um número maior de estudos e o fato de utilizar-se exclusivamente da base de dados da Biblioteca Virtual em Saúde. Sugere-se, portanto, a ampliação dessa busca, envolvendo um número maior de descritores e bases de dados, como Scopus, possibilitando dimensionar a contribuição que tais estudos têm trazido para o conhecimento científico na área da saúde coletiva com ênfase na saúde bucal.

\section{Referências}

AMORIM, K. P. C. et al. The construction of knowledge in Dentistry: the scientific production of three Brazilian magazines from 1990 to 2004. Interface, Botucatu, v. 11, n. 21, p. 9-23, Jan./Apr. 2007.

ANDERSSON, K. et al. The district nurse's perceptions of elderly patients' oral health: a qualitative interview study. Acta Odontol. Scand., Oslo, v. 65, no. 3, p. 177-182, June 2007.

ANDRADE, K. M. et al. Consensos em Saúde Bucal aprimorando a Atenção Básica, Ribeirão Preto, SP. Medicina, Ribeirão Preto, v. 44, n. 4, p. 355-366, 2011.

BEDOS, C. et al. How health professionals perceive and experience treating people on social assistance: a qualitative study among dentists in Montreal, Canada. BMC Health Serv. Res., London, v.13, p. 464-472, 2013.

BEDOS, C.; LEVINE, A.; BRODEUR, J. M. How people on social assistance perceive, experience, and improve oral health. J. dent. res., Washington, v. 88, no. 7, p. 653-657, July 2009.

BOGDAN, R. C.; BIKLEN, S. K. Qualitive research for education: an introduction for theory and methods. 3. ed. Boston: Allyn and Bacon, 1998.

BOSI, M. L. M. Pesquisa qualitativa em saúde coletiva: panorama e desafios Qualitative research in collective health: overview and challenges. Ciênc. Saúde Colet., Rio de Janeiro, v. 17, n. 3, p. 575586, 2012. 
BOWER, E; SCAMBLER, S. The contributions of qualitative research towards dental public health practice. Community dent. oral epidemiol., Copenhagen, v. 35, p. 161-169, 2007.

BRADBURY-JONES, C. et al. Dental neglect as a marker of broader neglect: a qualitative investigation of public health nurses' assessments of oral health in preschool children. BMC Public Health, London, v. 13, p. 370-381, 2013.

BRAVO, M. et al. Basic oral health goals for Spain 2015/2020. Int. dent. J., London, v.59, no.2, p.78-82, Apr. 2009.

CANE, R; WALKER, J. Rural public dental practice in Australia: perspectives of Tasmanian government-employed dentists. Aust. J. rural health, Armidale, v. 15, no. 4, p. 257-263, Aug. 2007.

CANESQUI, A. M. Produção científica das ciências sociais e humanas em saúde e alguns significados. Saúde Soc., São Paulo, v. 21, n. 1, p. 15-23, 2012.

CASHMORE, A.W. et al. Reorienting a pediatric oral health service towards prevention: lessons from a qualitative study of dental professionals. Health promot. J. Austr., West Perth, v. 22, no. 1, p. 17-21, Apr. 2011.

CASTAÑEDA, $H$. et al. False hope: effects of social class and health policy on oral health inequalities for migrant farmworker families. Soc. Sci. med., Oxford, v. 71, no. 11, p. 2028-2037, Dec. 2010.

CAVACA A.G. et al. As representações da saúde bucal na mídia impressa. Interface, Botucatu, v. 16, n. 43, p.1055-1068, 2012.

CELESTE, R. K.; WARMLING, C. M. Produção bibliográfica brasileira da Saúde Bucal Coletiva em periódicos da saúde coletiva e da odontologia. Ciênc. Saúde Colet., Rio de Janeiro, v.19, n.4, p. 19211932, 2014.

CELESTE, R. K.; BROADBENT, J. M.; MOYSES, S. J. Half-century of Dental Public Health research: bibliometric analysis of world scientific trends. Community dent. oral epidemiol., v. 44, n. 6, p. 557-563, 2016.

CORREIA, S. M. B; SILVEIRA J. L. G. C. percepção da relação saúde bucal e parto prematuro entre membros da equipe de ESF e gestantes. Pesqui. Bras. Odontopediatria clín. Integr., João Pessoa, v. 11, n. 3, p. 347-355, Jul./set. 2011.

COSTA, E. B. et al. Dor de dente: percepção dos usuários da atenção básica de saúde. Odontol. Clín. Cient., Recife, v. 8, n. 1, p. 53-58, 2009.

CLOVIS, J. B. et al. Using interviews to construct and disseminate knowledge of oral health policy. Int. j. dent. hyg., Oxford, v. 10, no. 2 , p. 91-97, May 2012.

CYRINO, A. P. Usos e abusos da pesquisa qualitativa em saúde em análise. Interface, Botucatu, v. 18, n. 50, p. 447, 2014.

DE MARCHI, R. J. et al. Vulnerability and the Psychosocial Aspects of Tooth Loss in Old Age: A Southern Brazilian Study. J. Cross Cult.

Gerontol., Dordrecht, v. 27, p. 239-258, 2012.

DENZIN, N. K.; LINCOLN, Y. S. Introdução: a disciplina e a prática da pesquisa qualitativa. In: LINCOLN, Y. S. et al. O planejamento da pesquisa qualitativa: teorias e abordagens. 2. ed. Porto Alegre. ArtMed, 2006. Cap. 1, p. 15-41.

DIAS, A. A.; NARVAI, P. C., RÊGO, D. M. Tendências da produção científica em odontologia no Brasil. Rev. Panam. salud pública, Washington, v. 24, n. 1, p.54-60, 2008.

DOLLINS, H. E.; BRAY, K. K.; GADBURY-AMYOT, C. C. A qualitative case study of the legislative process of the hygienist-therapist bill in a large midwestern state. J. Dent. Hyg., Chicago, v. 87, no. 5, p. 275288, Oct. 2013

DYER, T. A.; ROBINSON, P. G. General health promotion in general dental practice - the involvement of the dental team Part 2: A qualitative and quantitative investigation of the views of practice principals in South Yorkshire. Br. dent. j., London, v. 201, no. 1, p. 4551 , July 2006.

GALLAGHER, J.; CLARKE, W.; WILSON, N. Understanding the motivation: a qualitative study of dental students' choice of professional career. Eur. J. dent. educ., Copenhagen, v. 12, no. 2, p. 89-98, May 2008.

GARBIN, C. A. S. et al. Qualitative methodology: revealing a new path for research in Dentistry. Rev. gauch. odontol, Porto Alegre, v. 61, n. 3, p. 375-382, July./Set. 2013.

GEORGE, R.; KRUGER, E.; TENNANT, M. Qualitative research and dental public health. Indian j. dent. Res., Ahmedabad, v. 23, no. 1, p. 92-96, Jan. 2012.

GONÇALVES, E. R.; VERDI, M. I. M. Os problemas éticos no atendimento a pacientes na clínica odontológica de ensino. Ciênc. Saúde Colet., Rio de Janeiro, v. 12, n. 3, p.755-764, 2007.

GONÇALVES, H.; MENASHE, R. Pesquisando na interface: problemas e desafios a partir da pesquisa qualitativa em saúde. Interface, Botucatu, v. 18, n. 50, p. 449-456, 2014.

GONTIJO, L. P. T. et al. A saúde bucal coletiva na visão do estudante de odontologia - análise de uma experiência. Ciência \& Saúde Coletiva, Rio de Janeiro, v. 14, n. 4, p. 1277-1285, 2009.

GRANT, E. et al. The experiences of young oral cancer patients in Scotland: symptom recognition and delays in seeking professional help. Br. Dent. j., London, v. 208, no. 10, p. 465-471, May 2010.

GREENHALGH, T. et al. An open letter to the BMJ editors on qualitative research. BMJ, cidade, v. 352, p. i563, 2016.

HANCOCK, M.; CALNAN, M.; MANLEY, G. Private or NHS General Dental Service care in the United Kingdom? A study of public perceptions and experiences. Journal of Public Health Medicine, Oxford, v. 21, no. 4, p. 415-420, Dec. 1999.

HARRIS, R.V. et al. The use of conversation mapping to frame key perceptual issues facing the general dental practice system in England. Community dent. health., London, v. 26, no. 2, p. 84-91, June 2009.

HASTINGS G. B. et al. The role of the public in water fluoridation: public health champions or anti-fluoridation freedom fighters? $\mathbf{B r}$. Dent. j., London, v. 184, no. 1, p. 39-41, Jan. 1998. 
HOLMES, R. D. et al. Managing resources in NHS dentistry: the views of decision-makers in primary care organizations. Br. Dent. j., London, v. 205 , no. 6 , p. 328-329, Sept. 2008.

JACK, S. M. Utility of qualitative research findings in evidence- based public health practice. Public health nurs., Cambridge, v. 23, no. 3, p. 277-283, 2006.

KATEEB, E. Evaluation of the Ministry of Health school oral health programme in the West Bank region of Palestine. East. Mediterr. health j., Alexandria, v. 13, no. 3, p. 595-607, May/June 2007.

KNAUTH, D. R.; LEAL, A. F. A expansão das Ciências Sociais na Saúde Coletiva. Interface, Botucatu, v. 18, n. 50, p. 457-467, 2014.

KOYASHIKI, G. A. K.; SOUZA, R. A. A.; GARANHANI, M. L. O trabalho em saúde bucal do Agente Comunitário de Saúde em Unidades de Saúde da Família. Ciênc. Saúde Colet., Rio de Janeiro, v. 13, n. 4, p. 1343-1354, 2008.

LEE, M. A.; HORAN, S. A. Children's access to dental care in Connecticut's Medicaid managed care program. Matern. child health j., New York, v. 5, no. 1, p. 43-51, Mar. 2001.

LENZI T. L. et al. Perfil dos cirurgiões dentistas integrantes do Programa Saúde da Família em um município do Sul do Brasil. J. Health Sc. Inst., São Paulo, v. 28, n. 2, p. 121-124, 2010.

LEWIS, C. W.; LINSENMAYER, K. A.; WILLIAMS, A. Wanting better: a qualitative study of low-income parents about their children's oral health. Pediatr. dent., Chicago, v. 32, no. 7, p. 518-524, Nov./Dec. 2010.

LUZ, M. T.; MATTOS, R. S. Dimensões qualitativas na produção científica, tecnológica e na inovação em Saúde Coletiva. Ciênc. Saúde Colet., Rio de Janeiro, v. 15, n. 4, p. 1945-1953, 2010.

MASOOD M. et al. An appraisal of the quality of published qualitative dental research. Community dente. oral epidemiol., Copenhagen, v. 39, p. 193-203, 2011.

MATOS, P. E. S.; TOMITA, N. E. A inserção da saúde bucal no Programa Saúde da Família: da universidade aos pólos de capacitação. Cad. Saúde Pública, Rio de Janeiro, v. 20, n. 6, p. 1538-1544, 2004.

MCDONALD, R. et al. Changes to financial incentives in English dentistry 2006-2009: a qualitative study. Community dente. oral epidemiol., Copenhagen, v. 40, no. 5, p. 468-473, Oct. 2012.

MCKEOWN, L. Social relations and breath odour. Int. j. dent. hyg., Oxford, v. 1, no. 4, p. 213-217, Nov. 2003.

MIGUEL, L. C.; REIBNITZ JÚNIOR C.; PRADO M. L. Pesquisa quantitativa: um outro caminho para a produção do conhecimento em odontologia. Rev. ABENO, Brasília, v. 1, p. 130-134, 2007.

MINAYO, M. C. S. O desafio do conhecimento: pesquisa qualitativa em saúde. 12. ed. São Paulo: Hucitec, 2010.

MINAYO, M. C. S. Análise qualitativa: teoria, passos e fidedignidade. Ciênc. Saúde Colet.,Rio de Janeiro, v. 17, n. 3, p. 621-626, 2012.
MUNKEVIZ, M. S. G.; PELICIONI M. C. F. Saúde bucal na estratégia saúde da família no município de São Paulo: perspectiva do usuário. Rev. br. cresc. desenvol. hum., [S. I.], v. 20, n. 3, p. 791-801, 2010.

NADANOVSKY, P. O aumento da produção científica odontológica brasileira na saúde pública. Cad. Saúde Pública, Rio de Janeiro, v. 22 , n. 5, p. 886-887, 2006.

NASCIMENTO, A. C. et al. Oral health in the family health strategy: a change of practices or semantics diversionism. Rev. Saúde Pública, São Paulo, v. 43, n. 3, p. 455-462, 2009.

NATIONS, M. K. et al. Cultural significance of primary teeth for caregivers in Northeast Brazil. Cad. Saúde Pública, Rio de Janeiro, v. 24 , n. 4, p. 800-808, 2008.

NEVES, M.; PRETTO, S. M.; ELY, H. C. Percepções de usuários e trabalhadores de saúde sobre a implantação do acolhimento em uma unidade de saúde em Porto Alegre-RS, Brasil. Rev. odontol. UNESP, Marília, v. 42, n. 5, p. 364-371, 2013.

NEWTON, T. Qualitative research and evidence-based dentistry: linking evidence to practice. Evidenc. based dente., [S. I.], v. 2, no. 4 , p. 104-106, 2000.

NUTO, S. A. S.; NATIONS, M. K.; COSTA, I. C. C. Aspectos culturais na compreensão da periodontite crônica: um estudo qualitativo. Cad. Saúde Pública, Rio de Janeiro, v. 23, n. 3, p. 681-690, 2007.

PANG, L. C.; ANDERSON, V. R.; MOFFAT, S. M. Initial University of Otago oral health graduates' employment pathways and preparedness for work. N. Z. dent. j., Dunedin, v. 108, no. 3, p. 83-88, Sept. 2012.

PEREIRA, D. Q.; PEREIRA, J. C. M.; ASSIS, M. M. A. A prática odontológica em Unidades Básicas de Saúde em Feira de Santana (BA) no processo de municipalização da saúde: individual, curativa, autônoma e tecnicista. Ciênc. Saúde Colet., Rio de Janeiro, v. 8, n. 2, p. 599-609, 2003.

PEZZATO, L.M.; L'ABBATE, S. O uso de diários como ferramenta de intervenção da análise institucional: potencializando reflexões no cotidiano da Saúde Bucal Coletiva. Physis, Rio de Janeiro, v. 21, n. 4, p. 1297-1314, 2011.

PEZZATO, L.M.; L'ABBATE, S. Uma pesquisa-ação-intervenção em Saúde Bucal Coletiva: contribuindo para a produção de novas análises. Saúde Soc., São Paulo, v. 21, n. 2, p. 386-398, 2012.

PINE, C. M.; DUGDILL, L. Analysis of a unique global public-private partnership to promote oral health. Int. dent j., London, v. 61 Suppl. 2, p. 11-21, Aug. 2011.

PINELLI, C. et al. Biossegurança e odontologia: crenças e atitudes de graduandos sobre o controle da infecção cruzada. Saúde Soc., São Paulo, v. 20, n. 2, p. 448-461, 2011.

POPE, C.; MAYS, N. Métodos qualitativos na pesquisa em saúde. In: . Pesquisa qualitativa na atenção à saúde. 3 . ed. Porto Alegre: Artmed, 2009. Cap. 1, p. 11-21.

PRZYLYNSKI, D. S. et al. Ações educativas de enfermagem em saúde bucal de idosos em uma instituição de longa permanência. Cogitare enferm., Curitiba, v. 14, n. 4, p. 696-702, out./dez. 2009. 
REIBNITZ JÚNIOR, C.; CAETANO, J. C.; PRADO, M. L. A contribuição do trabalho odontológico na resolução de problemas de saúde da população: a concepção de alunos de Odontologia. Physis, Rio de Janeiro, v. 19, p. 189-206, 2009.

RODRIGUES, A. A. A. O.; BOMFIM, L. S. Saúde bucal no programa saúde da família em município do semiárido baiano (Feira de Santana): organização e micropolítica. Ver. APS, Juiz de Fora, v. 13, n. 1, p. 96-108, jan./mar. 2010.

SANTIAGO, W. K.; WERNECK, M. A. F.; FERREIRA, E. F. O processo de inserção da Odontologia no PSF de Belo Horizonte/MG: um estudo qualitativo com base na visão da equipe de coordenação de saúde bucal e cirurgiões dentistas do programa saúde da família.

Arq. Odontol.., Belo Horizonte, v. 45, n. 4, p. 211-219, out./dez. 2009.

SERAPIONI, M. Métodos qualitativos e quantitativos na pesquisa social em saúde: algumas estratégias para a integração. Ciênc.

Saúde Colet., Rio de janeiro, v. 5, n. 1, p. 187-192, 2000.

SILVA, M. L.; GOMES FILHO, D. L. A percepção dos usuários sobre a incorporação da equipe de saúde bucal no programa de saúde da família. Pesq. bras. odontopediatria clín. integr., João Pessoa, v. 12, n. 3, p. 369-376, jul./set. 2012.

SOARES, C. L. M.; PAIM, J. S. Aspectos críticos para a implementação da política de saúde bucal no município de Salvador, Bahia, Brasil. Cad. Saúde Pública, Rio de Janeiro, v. 27, n. 5, p. 966974, 2011.

STEWART, K. et al. Qualitative research in dentistry. Br. dent. j., London, v. 204, no. 5, p. 235-239, 2008.

STOTO, M.; NELSON, C.; KLAIMAN, T. Getting from what to why: using qualitative research to conduct public health systems research. Trabalho apresentado no Academy Health's 2012 PHSR Interest Group Annual Meeting, Orlando, Flórida.

TEIXEIRA, J. A. et al. Prevenção de oclusopatias em saúde pública: realidade ou utopia? Ortho Sci., Orthod. Sci. pract., Curitiba, v. 1, n. 1, p. 48-53, 2008.

TITCHEN, A.; HOBSON, D. Compreensão da fenomenologia mediante perspectivas inversas. In: SOMEKH, B.; LEWIN, C. (Orgs.). Teoria e método de pesquisa social. Rio de Janeiro: Vozes, 2015. p.183-191.

TOASSI, R. F. C. et al. O ensino nos serviços de atenção primária do Sistema Único de Saúde (SUS) na formação de profissionais de saúde no Brasil. Interface, Botucatu, v. 17, n. 45, p. 385-92, abr./jun. 2013.

TURATO, E. R. Métodos qualitativos e quantitativos na área da saúde: definições, diferenças e seus objetos de pesquisa. Rev. Saúde Pública, São Paulo, v. 39, n. 3, p. 507-514.

UMUNNA, A. O.; JAMES, V. S.; RICKS, E. J. The experiences of dentists in the management planning of oral health services in Lesotho, Africa. Rural remote health., Geelong, v. 9, no. 4, p. 1213, Oct./Dec. 2009.

VARGAS, A. M. D.; PAIXÃO, H. H. Perda dentária e seu significado na qualidade de vida de adultos usuários de serviço público de saúde bucal do Centro de Saúde Boa Vista, em Belo Horizonte. Ciênc.

Saúde Colet., Rio de Janeiro, v. 10, n. 4, p. 1015-1024, 2005.
VASCONCELOS, M.; CARDOSO, A. V. L.; ABREU, M. H. N. G. Os desafios dos agentes comunitários de saúde em relação à saúde bucal em município de pequeno porte. Arq. odontologia, Belo Horizonte, v. 46, n. 2, p. 98-104, abr./jun. 2010.

WALLACE, B. B.; MACENTEE, M. I. Access to dental care for lowincome adults: perceptions of affordability, availability and acceptability. J. commun. Health., New York, v. 37, no. 1, p. 32-39, Feb. 2012. 